\title{
Mejora de las concepciones de maestros en formación de la educación STEM
}

\author{
Radu Bogdan Toma ${ }^{1}$ \\ Diego Armando Retana-Alvarado ${ }^{2}$ \\ ${ }^{1}$ Universidad de Burgos (UBU), España; ${ }^{2}$ Universidad de Costa Rica (UCR), Costa Rica.
}

Resumen. El creciente interés por el acrónimo STEM ha dado lugar a la coexistencia de diversas conceptualizaciones que desafían su comprensión. En la medida en que se pretenda que estas siglas -recientemente definidas como un enfoque educativo- supongan un avance significativo en la didáctica de las ciencias, resulta necesario mejorar la comprensión de los docentes sobre su significado. Este estudio presenta un programa de formación que tiene por objetivo tal esfuerzo, y presenta los resultados de su implementación con profesorado de ciencias costarricenses en formación inicial. Se ha identificado una multiplicidad de conceptualizaciones que han conformado seis modelos STEM distintos desde simplistas (p.ej. STEM como un mero acrónimo) hasta desarrollados y coherentes con las definiciones actuales (p.ej. STEM como la integración curricular de cuatro disciplinas). Al inicio del programa han predominado las conceptualizaciones de STEM como cuatro disciplinas separadas o STEM como un acrónimo. La intervención ha permitido avanzar en el abandono de estas conceptualizaciones simplistas. Sin embargo, se han detectado dificultades en el desarrollo de conceptualizaciones coherentes con las visiones actuales de la educación STEM (p.ej. STEM como conexión o integración curricular de cuatro disciplinas), lo que plantea dudas sobre la idoneidad de un enfoque STEM integrado.

Palabras clave: conceptualización; modelo educacional; educación intercultural; didáctica; formación de docentes; STEM.

\section{Melhoria das concepções dos professores estagiários sobre a educação STEM}

Resumo. O crescente interesse pela sigla STEM levou à coexistência de diversas conceitualizações que desafiam a sua compreensão. Na medida em que esta sigla - recentemente definida como uma abordagem educacional - pretende representar um avanço significativo na didática das ciências, é necessário melhorar a compreensão dos professores sobre o seu significado. Este estudo apresenta um programa de formação visando esse esforço e os resultados da sua implementação junto aos professores de Ciências costarriquenhos na sua formação inicial. Identificou-se uma multiplicidade de conceitos que estabeleceram seis modelos diferentes de STEM, desde modelos simplistas (por exemplo, STEM como um mero acrônimo) até os desenvolvidos e consistentes com as definições atuais (por exemplo, STEM como a integração curricular de quatro disciplinas). No início do programa, predominaram as conceitualizações da STEM como quatro disciplinas separadas ou STEM como acrônimo. A intervenção conseguiu avançar no sentido de se afastar desses conceitos simplistas. No entanto, identificaram-se dificuldades no desenvolvimento de conceitos coerentes com a visão atual da educação STEM (por exemplo, STEM como uma conexão ou integração curricular de quatro disciplinas), levantando dúvidas sobre a idoneidade de uma abordagem integrada STEM.

Palavras-chave: conceitualização; modelo educacional; educação intercultural; didática; formação de professores; STEM.

\section{Improving pre-service teachers' conceptions of STEM education}

Abstract. The growing interest for STEM acronym has resulted in diverse conceptualizations coexisting which challenge its understanding. As long as this acronym -recently defined as an educational approach-is intended to produce progress in science education, there is a need to improve teachers' understanding about its meaning. This study presents a professional development program that aims at such an endeavor, and presents the results of its implementation with Costa Rican pre-service science teachers. A myriad of different conceptualizations have been identified, resulting in a six different STEM models ranging from the simplest (e.g. STEM as a mere acronym) to more developed models consistent with current definitions (e.g. STEM as the curricular integration of four disciplines). At the start of the program, conceptualizations of STEM as four separate disciplines or STEM as an acronym prevailed. The intervention has led to progress in abandoning simplistic conceptualizations. However, difficulties have been identified in developing conceptualizations consistent with current views of STEM education (e.g. STEM as a connection or curricular integration of four disciplines) raising questions about the appropriateness of an integrated STEM approach.

Keywords: conceptualization; educational model; intercultural education; didactics; teacher training; STEM. 


\section{Introducción}

El acrónimo STEM se ha convertido en objeto de atención internacional para las reformas curriculares de la enseñanza de las ciencias, la tecnología, la ingeniería y las matemáticas. Sin embargo, a medida que su uso ha traspasado la esfera política y se ha extendido en el panorama educativo, STEM se han empleado de forma imprecisa, dando lugar a una amplia variedad de definiciones (Margot y Kettler, 2019; Martín-Páez et al., 2019). Por lo tanto, STEM se presenta principalmente con tres significados diferentes (Toma y García-Carmona, 2021): (i) como un acrónimo de origen político que demanda una mayor atención administrativa (en términos de financiación y mejora curricular) hacia las disciplinas que lo componen; (ii) como un eslogan para comercializar un amplio abanico de productos, tales como conferencias educativas, libros, o materiales didácticos varios; y (iii) más recientemente, STEM como un movimiento pedagógico que promueve un plan de estudios integrado o interdisciplinar, lo que se materializa como educación STEM integrada (Johnson et al., 2016; Kelley y Knowles, 2016).

La educación STEM integrada también se ha definido de forma imprecisa, con concepciones diversas que varían desde la visión de STEM como una sola materia o disciplina hasta posturas que conciben STEM como un enfoque completamente transdisciplinar (Bybee, 2013; Ring et al., 2017). De este modo, una de las definiciones más comunes de la educación STEM integrada es la de un enfoque que "(...) explora la enseñanza y el aprendizaje entre dos o más de las áreas temáticas STEM, y/o entre una materia STEM y una o más materias escolares" (Sanders, 2009, p. 21). Por lo tanto, esta perspectiva implica concebir las disciplinas STEM como una unidad que se ha de enseñar de manera integrada y cohesiva (Breiner et al., 2012; Toma y Greca, 2018).

En este sentido, a nivel internacional, la educación STEM se suele traducir como el uso pedagógico del diseño ingenieril para explorar tecnologías y, de este modo, aplicar conocimientos matemáticos y científicos (Johnson et al., 2016). Sin embargo, en el contexto español algunos autores conciben STEM como una amplia amalgama de enfoques y estrategias didácticas. Bajo la premisa de que "(...) lo honesto y lo práctico, más que intentar dotar a STEM de un significado didáctico o metodológico per se, sería considerar STEM todo lo que pueda sumar a la consecución de esos objetivos políticos" (Domènech-Casal, 2019, p. 157), STEM se define como "(...) distintos enfoques metodológicos, herramientas tecnológicas y perspectivas educativas precedentes o de nuevo cuño" (p. 157), o como "(...) una oportunidad de trabajar, de forma más o menos integrada, las formas de pensar, hacer hablar, valorar y ser propias del ámbito científico-tecnológico (Couso y Grimalt-Álvaro, 2020, p. 72). Así, se incluyen bajo el paraguas STEM desde la impresión $3 D$ hasta abordajes como la perspectiva Ciencia-Tecnología-Sociedad (CTS), pasando por todo tipo de enfoques didácticos, tales como la resolución de problemas, la indagación, la modelización, el design thinking, el fenómeno maker, el tinkering, o el pensamiento computacional, entre muchos otros aspectos de difícil justificación (p.ej. Couso y Grimalt-Álvaro, 2020; Domènech-Casal, 2019). 
En definitiva, se observa que una definición consensuada de la educación STEM permanece esquiva, lo que supone una primera barrera para la adopción de un enfoque de estas características. Diversas investigaciones han comprobado que el profesorado posee concepciones muy limitadas y diversas de aquello que constituye STEM (Margot y Kettler, 2019; Radloff y Guzey, 2016; Ring et al., 2017). Dado que las concepciones docentes repercuten en las prácticas pedagógicas, es fundamental desarrollar programas de formación enfocados a superar conceptualizaciones erróneas sobre la educación STEM (Honey et al., 2014; Roehrig y Luft, 2004). Sin ello, la probabilidad de que este enfoque se adopte de manera eficaz es escasa.

Por ello, el propósito de este estudio fue explorar las conceptualizaciones de un grupo de maestros costarricenses en formación inicial (MCFI en adelante) sobre la educación STEM antes y después de participar en un programa STEM de desarrollo profesional de seis semanas de duración, implementado de manera virtual por las restricciones provocadas por la pandemia de COVID-19. Se formularon dos preguntas de investigación:

i ¿Cómo conceptualizan los MCFI la educación STEM?

ii ¿De qué manera cambia la conceptualización de los MCFI después de participar en un programa STEM de formación inicial?

\section{Métodos y materiales}

\subsection{Programa de formación}

El programa de formación fue diseñado siguiendo recomendaciones contemporáneas (Capps et al., 2012) con el objetivo de identificar y mejorar las concepciones de los docentes en formación sobre la educación STEM, y capacitarles en el uso de dos estrategias pedagógicas (indagación escolar y diseño ingenieril) para su implementación. El programa fue implementado en el marco de la asignatura FD0516 Ciencias en la Educación Primaria II, del segundo curso del Grado Bachillerato en Educación Primaria de la Universidad de Costa Rica. Esta asignatura de tres créditos (3 horas teóricas y 6 horas prácticas cada semana - 144h totales por semestre) facilita el desarrollo de un Conocimiento Didáctico del Contenido (CDC) sobre la materia y sus transformaciones mediante el abordaje de las ideas científicas clave, las representaciones del contenido, prácticas científicas y estrategias de evaluación que permiten superar obstáculos afectivos y cognitivos (p.ej. emociones negativas o ideas alternativas) en el aprendizaje de los contenidos establecidos en el programa de Ciencias del Ministerio de Educación Pública de Costa Rica. Dichos elementos curriculares se integran desde la metodología indagatoria, empleándose la plataforma Mediación Virtual como entorno digital de interacción entre el profesor y los estudiantes.

El programa se implementó de manera virtual debido a las medidas de confinamiento domiciliario derivadas de la pandemia del COVID-19, a razón de seis semanas con una sesión sincrónica (90 minutos) y tareas asincrónicas individuales y grupales (60-90 minutos) cada semana (Tabla 1)1. Específicamente, el programa se basó en el modelo de educación STEM propuesto por Toma (2020b), que define la educación

\footnotetext{
${ }^{1}$ Cabe resaltar que la intervención se diseñó considerando las limitaciones ocasionadas por el contexto COVID-19, en el que, por motivos logísticos, se redujo el número de horas prácticas semanales. Durante la intervención, los participantes no realizaron otras actividades docentes relacionadas con la asignatura más allá de las recogidas en la Tabla 1.
} 
STEM como " (...) un paradigma educativo dirigido a reducir intencionadamente las fronteras entre la Ciencia, la Tecnología, la Ingeniería y las Matemáticas, fomentando episodios holísticos de enseñanza y aprendizaje basados en auténticos problemas del mundo real, en los que se valora y reflexiona explícitamente sobre las similitudes, las diferencias y la relación entre los conceptos, los procedimientos y las habilidades de cada una de estas disciplinas (p. 13).

Este modelo se caracteriza por el planteamiento de una situación problemática curricularmente relevante y socialmente importante (p.ej. calidad del agua) cuya resolución requiere de dos fases: (i) aprendizaje de conocimientos científicos y desarrollo de habilidades científicas que permitan entender y abordar la situación problemática planteada, mediante distintas indagaciones (p.ej. parámetros físico-químicos de la calidad del agua); y (ii) aplicación de los conocimientos adquiridos al diseño, desarrollo, evaluación y mejora, empleando el proceso de diseño ingenieril, de un producto o proceso tecnológico que ayude a solucionar el problema formulado (p.ej. un filtro depurador de agua). A lo largo de ambas fases, se lleva a cabo una reflexión explícita y crítica sobre la forma en que operan las disciplinas STEM en la vida real para la resolución de problemas y el desarrollo de nuevos conocimientos. Un ejemplo detallado de este modelo, así como de su transposición didáctica puede consultarse en Toma (2020a).

Tabla 1. Programa de formación inicial

\begin{tabular}{|c|c|c|c|}
\hline $\begin{array}{c}\text { Sema- } \\
\text { nas }\end{array}$ & $\begin{array}{l}\text { Módu- } \\
\text { los }\end{array}$ & Sesión sincrónica & Tareas asincrónicas \\
\hline $\begin{array}{l}1^{\mathrm{a}} \\
\text { sema- } \\
\text { na }\end{array}$ & $\begin{array}{l}\text { :응 } \\
\text { 을 } \\
\text { 을 } \\
\stackrel{\underline{L}}{\underline{E}}\end{array}$ & $\begin{array}{l}\text { - Reseña histórica al acrónimo STEM } \\
\text { - Recogida de datos (pretest) } \\
\text { - Introducción a STEM: tipos de enfoques STEM } \\
\text { (Bybee, 2013) } \\
\text { - Modelo pedagógico interdisciplinar (Toma, } \\
\text { 2020b) }\end{array}$ & $\begin{array}{l}\text { Lectura y recensión crítica } \\
\text { del artículo ¿Qué es STEM } \\
\text { y STEAM (Couso y Grimalt- } \\
\text { Álvaro, 2020) }\end{array}$ \\
\hline $\begin{array}{l}2^{\mathrm{a}} \\
\text { sema- } \\
\text { na }\end{array}$ & $\frac{\sqrt{0}}{\frac{0}{2}}$ & $\begin{array}{l}\text { - Definición indagación científica (Crawford, } \\
\text { 2014) } \\
\text { - El continuum de indagaciones (Martin-Hansen, } \\
\text { 2002) } \\
\text { - Fases de una indagación (Pedaste et al., 2015) } \\
\text { - Introducción indagación confirmatoria: "Roza- } \\
\text { miento" } \\
\text { https://bit.ly/3sLZUPH }\end{array}$ & $\begin{array}{l}\text { - Lectura y recensión } \\
\text { crítica del artículo La } \\
\text { Indagación Científica: un } \\
\text { concepto esquivo, pero } \\
\text { necesario (Vergara y } \\
\text { Cofré, 2012) } \\
\text { - Desarrollo de la inda- } \\
\text { gación "Rozamiento" de } \\
\text { manera grupal }\end{array}$ \\
\hline $\begin{array}{l}3^{a} \\
\text { sema- } \\
\text { na }\end{array}$ & 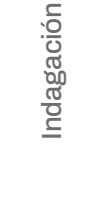 & $\begin{array}{l}\text { - Presentación y puesta en común de la indaga- } \\
\text { ción "Rozamiento" } \\
\text { • Introducción indagación estructurada: "Oxida- } \\
\text { ción" } \\
\text { https://bit.ly/3zjfnsF }\end{array}$ & $\begin{array}{l}\text { - Desarrollo de la inda- } \\
\text { gación “Oxidación" de } \\
\text { manera grupal }\end{array}$ \\
\hline $\begin{array}{l}4^{a} \\
\text { sema- } \\
\text { na }\end{array}$ & & $\begin{array}{l}\text { - Presentación y puesta en común de la indaga- } \\
\text { ción "Oxidación" } \\
\text { • Introducción indagación guiada: "El almuerzo" } \\
\text { https://bit.ly/3Bfcx8U }\end{array}$ & $\begin{array}{l}\text { - Desarrollo de la inda- } \\
\text { gación "El almuerzo" de } \\
\text { manera grupal }\end{array}$ \\
\hline
\end{tabular}




\begin{tabular}{|c|c|c|c|}
\hline $\begin{array}{c}\text { Sema- } \\
\text { nas }\end{array}$ & $\begin{array}{l}\text { Módu- } \\
\text { los }\end{array}$ & Sesión sincrónica & Tareas asincrónicas \\
\hline $\begin{array}{l}5^{\mathrm{a}} \\
\text { sema- } \\
\text { na }\end{array}$ & 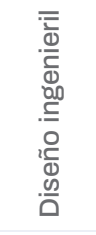 & $\begin{array}{l}\text { - Presentación y puesta en común de la indaga- } \\
\text { ción "El almuerzo de Carlos" } \\
\text { - Definición del Modelo de Diseño Ingenieril } \\
\text { - Introducción reto ingenieril "Egg drop challen- } \\
\text { ge": } \\
\text { https://bit.ly/3zlxlLd }\end{array}$ & $\begin{array}{l}\text { - Desarrollo del reto inge- } \\
\text { nieril "Egg drop challen- } \\
\text { ge" de manera grupal }\end{array}$ \\
\hline $\begin{array}{l}6^{a} \\
\text { sema- } \\
\text { na }\end{array}$ & 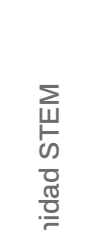 & $\begin{array}{l}\text { - Presentación y puesta en común del reto inge- } \\
\text { nieril "Egg drop challenge" } \\
\text { - Ejemplificación de la unidad didáctica STEM de } \\
\text { Toma (2020a) sobre los parámetros fisicoquími- } \\
\text { cos de la calidad del agua (indagación) y técni- } \\
\text { cas de separación de mezcla (reto ingenieril). }\end{array}$ & $\begin{array}{l}\text { - Diseño y desarrollo de } \\
\text { una unidad didáctica } \\
\text { STEM }\end{array}$ \\
\hline $\begin{array}{l}7^{\mathrm{a}} \\
\text { sema- } \\
\text { na }\end{array}$ & ऽั & $\begin{array}{l}\text { - Presentación y puesta en común de las unida- } \\
\text { des didácticas STEM diseñadas por el alumnado } \\
\text { - Recogida de datos (posttest) }\end{array}$ & \\
\hline
\end{tabular}

Fuente: elaboración propia.

De este modo, tres módulos distintos han constituido el programa de formación propuesto de esta investigación. En primer lugar, una introducción teórica al acrónimo STEM y a su transposición didáctica en la educación científica. A continuación, un módulo dedicado a aprender, diseñar y desarrollar un total de tres indagaciones escolares con distinto grado de andamiaje -confirmatoria, estructurada y guiada-según el continuum de Herron (1971) y Martin-Hansen (2002). Posteriormente, un módulo destinado a descubrir las fases del modelo de diseño ingenieril y a su uso de manera pedagógica mediante el diseño y desarrollo de un reto ingenieril fundamentado en Cunningham (2018). Finalmente, un módulo que establece conexiones entre las indagaciones y el reto ingenieril para conformar una unidad didáctica STEM. Durante la séptima semana, los participantes presentaron de manera virtual las unidades didácticas STEM que han debido diseñar. Este artículo se limita a explorar las concepciones de los MCFI sobre la educación STEM antes y después de la participación en el programa de formación. En trabajos futuros, se explorará la relación entre estas concepciones y su capacidad para diseñar unidades didácticas coherentes con la educación STEM integrada.

\subsection{Muestra}

Se conformó un muestreo de conveniencia a partir de 26 estudiantes matriculados. La gran mayoría de los participantes se identificaron con el sexo femenino (84.6\%) y la edad media del grupo fue de 20.8 años $(D E=4.14)$. En cuanto a su formación básica, 21 de los estudiantes cursaron un bachillerato de biología, y los 5 restantes un bachillerato de física. Por lo tanto, se puede considerar que todos los participantes poseen un adecuado nivel de conocimiento de contenido científico. Debido a las complicaciones causadas por la pandemia COVID-19, un participante ha fallado en enviar sus respuestas del pretest y tres participantes no han cumplimentado el posttest. Tras eliminar estos casos perdidos, la muestra válida para este estudio está compuesta por 22 estudiantes. 


\subsection{Instrumento}

Se ha empleado el protocolo de elicitación propuesto por Ring et al., (2017). Se pidió a los participantes que (i) representaran visualmente sus concepciones de la educación STEM mediante un dibujo (1. Realiza un dibujo que represente tu conceptualización de la educación STEM); (ii) explicaran y describieran su conceptualización (2. Explica y describe con palabras tu conceptualización de la educación STEM); y (iii) reflexionaran sobre cualquier experiencia que hubiera configurado su conceptualización (3. ¿Qué experiencias o conocimientos han influido en tu conceptualización de la educación STEM?).

Este protocolo se administró durante la primera y la última sesión del programa de formación. En cuanto a la primera sesión, el protocolo fue administrado tras una breve reseña histórica del acrónimo STEM, basada en Toma y García-Carmona (2021) y Bybee (2013). Se tomaron precauciones para no proporcionar ninguna conceptualización, definición, o crítica de STEM, sino únicamente los orígenes de este acrónimo. Durante la segunda administración, se devolvieron los modelos iniciales a cada participante para su reflexión personal; seguidamente se les animó a reflexionar sobre su primer modelo y a volver a dibujar y describir su conceptualización, indicando si seguía siendo la misma o si, por el contrario, había cambiado. En cada administración del protocolo, se informó a los participantes que no existe una única respuesta válida.

\subsection{Procedimiento y análisis}

Las respuestas de los participantes se analizaron mediante una combinación de técnicas de análisis de contenido cualitativo convencional y dirigido (Hsieh y Shannon, 2005). En la primera iteración, dos investigadores utilizaron análisis de contenido convencional, evitando así la imposición de conceptualizaciones STEM procedentes de la literatura. El análisis comenzó con una inspección de cada protocolo para desarrollar una comprensión del conjunto de los datos. A continuación, se derivaron distintos códigos que capturaban ideas centrales sobre STEM. Este proceso iterativo dio lugar un listado inicial de distintos modelos de educación STEM. La respuesta de tres participantes aludía a más de un modelo de educación STEM, por lo que se ha categorizado en aquella conceptualización que predominaba tanto en la respuesta escrita como en la representación. En la segunda iteración, los investigadores utilizaron el enfoque dirigido de análisis de contenido. Todos los protocolos se volvieron a analizar a la luz de los modelos STEM propuestos por Bybee (2013), Ring et al. (2017), y Dare et al. (2019), resumidos en la Tabla 2. De esta manera, se conformó un continuum de educación STEM que se empleó para categorizar las respuestas de los participantes en seis conceptualizaciones o modelos distintos, desde muy restringidos (STEM como un acrónimo) hasta congruentes con las definiciones existentes en la literatura (STEM como integración de cuatro disciplinas). Se calculó la concordancia interevaluador, obteniéndose un porcentaje de acuerdo del 90\% entre ambos investigadores. Las diferencias se resolvieron por consenso hasta alcanzar máximo acuerdo. 
Tabla 2. Modelos STEM empleados durante el análisis de contenido dirigido

\begin{tabular}{|c|c|c|c|}
\hline & Bybee (2013) & Ring et al., (2017) & Dare et al., (2019) \\
\hline $\mathrm{i}$ & Ciencia o matemáticas & Disciplinas integradas & Acrónimo \\
\hline ii & Ciencia y matemáticas & Ciencia como contexto & $\begin{array}{l}\text { Resolución de problemas } \\
\text { como contexto }\end{array}$ \\
\hline iii & $\begin{array}{l}\text { Ciencia que incorpora tec- } \\
\text { nología, ingeniería o mate- } \\
\text { máticas }\end{array}$ & $\begin{array}{l}\text { Diseño ingenieril como con- } \\
\text { texto }\end{array}$ & Ciencia como contexto \\
\hline iv & Disciplinas separadas & $\begin{array}{l}\text { Ciencia y diseño ingenieril } \\
\text { como contexto }\end{array}$ & Disciplinas separadas \\
\hline v & $\begin{array}{l}\text { Ciencias y matemáticas } \\
\text { conectadas por tecnología } \\
\text { o ingeniería }\end{array}$ & $\begin{array}{l}\text { Resolución de problemas } \\
\text { como contexto }\end{array}$ & Disciplinas integradas \\
\hline vi & $\begin{array}{l}\text { Coordinación entre disci- } \\
\text { plinas }\end{array}$ & Disciplinas separadas & $\begin{array}{l}\text { Diseño ingenieril como con- } \\
\text { texto }\end{array}$ \\
\hline vii & $\begin{array}{l}\text { Combinación de dos o tres } \\
\text { disciplinas }\end{array}$ & Acrónimo & $\begin{array}{l}\text { Ciencia y diseño ingenieril } \\
\text { como contexto }\end{array}$ \\
\hline viii & Disciplinas integradas & Ingeniería como contexto & Ingeniería como contexto \\
\hline xix & $\begin{array}{l}\text { Programa o curso transdis- } \\
\text { ciplinar }\end{array}$ & STEM y otras disciplinas & ----- \\
\hline$x$ & ---- & Trabajo en equipo & ---- \\
\hline xi & ---- & Comunicación & ----- \\
\hline
\end{tabular}

Fuente: elaboración propia.

\section{Resultados}

\section{1 ¿Cómo conceptualizan los MCFI la educación STEM?}

En primer lugar, a partir del análisis de contenido cualitativo de los datos previos y posteriores al programa de formación, se han identificado un total de seis modelos de educación STEM, representados en la Figura 1 atendiendo al grado de integración disciplinar. Estos modelos representan las conceptualizaciones de los participantes en este estudio.

El modelo A concibe STEM como un mero acrónimo que se emplea para hacer referencia a las disciplinas de Ciencia, Tecnología, Ingeniería y Matemáticas, sin que haya mención explícita a su enseñanza y aprendizaje (Figura 2):

La educación STEM incorpora en el estudiantado la ciencia, tecnología, ingeniería y matemáticas. Esto ayuda que todos los niños y las niñas puedan entender todos los avances y aportes impulsados por cada una de las disciplinas ya mencionadas. 
Modelo A

\begin{tabular}{|c|}
\hline Acrónimo \\
STEM \\
Science, Technology, \\
Engineering, Mathematics
\end{tabular}

Modelo D

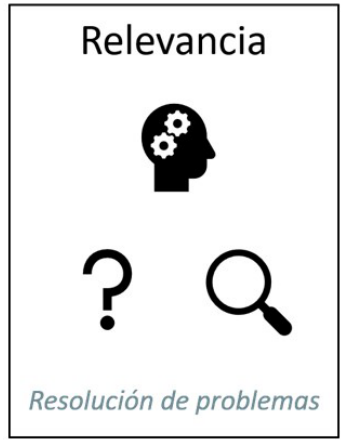

Modelo B

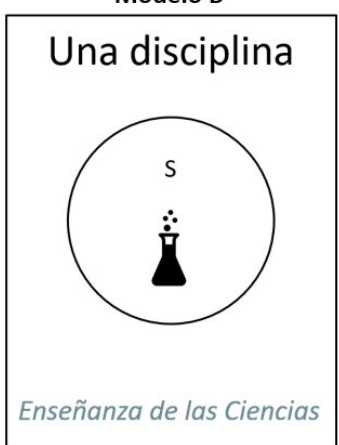

Modelo E

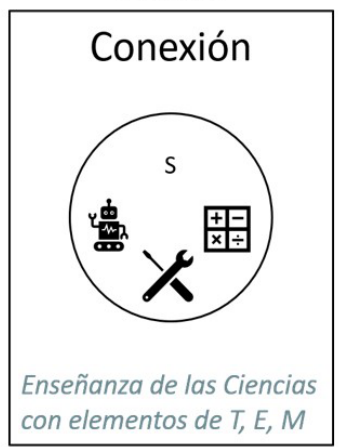

Modelo C

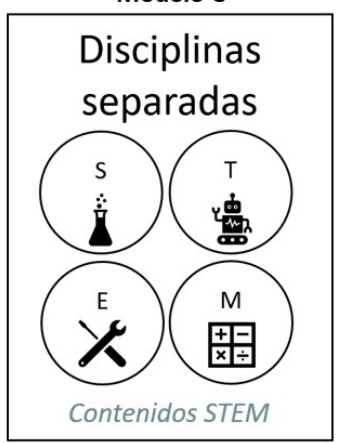

Modelo F

Integración

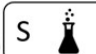

M 缃

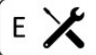

Integración de los contenidos STEM

Figura 1. Modelos STEM derivados a partir del análisis de contenido cualitativo. Fuente: archivo de los autores.

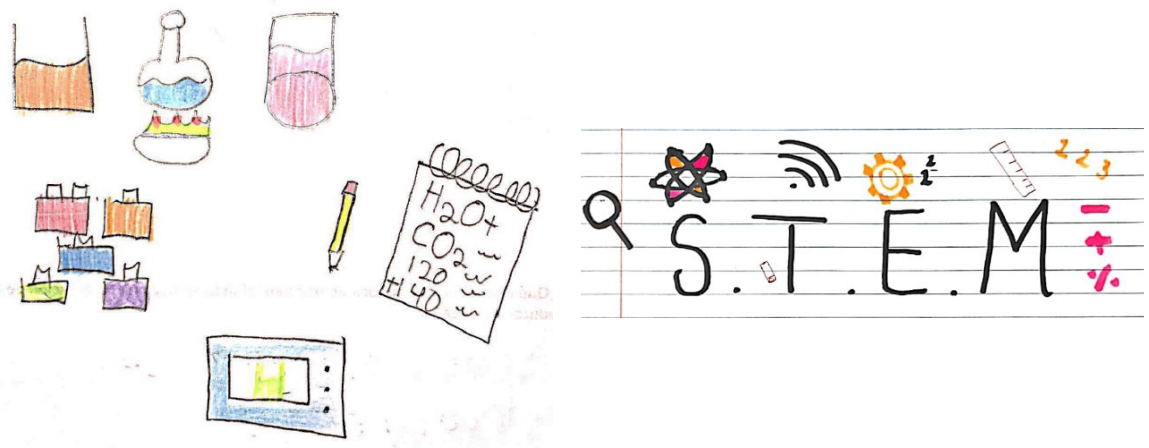

Figura 2. Ejemplo de representaciones categorizadas como el modelo A de educación STEM.

Fuente: archivo de los autores.

El modelo B representa STEM como una única disciplina, generalmente la científica. Según esta conceptualización, STEM es sinónimo de enseñanza de las ciencias, con referencias explícitas a innovaciones didácticas (Figura 3): 
"La educación STEM es una tendencia educativa en la que se integran muchos conceptos educativos como el constructivismo y el conectivismo."

"La educación STEM es la implementación de diferentes metodologías en el aula. Esto con el fin de que todos los estudiantes desarrollen habilidades científico-tecnológicas."

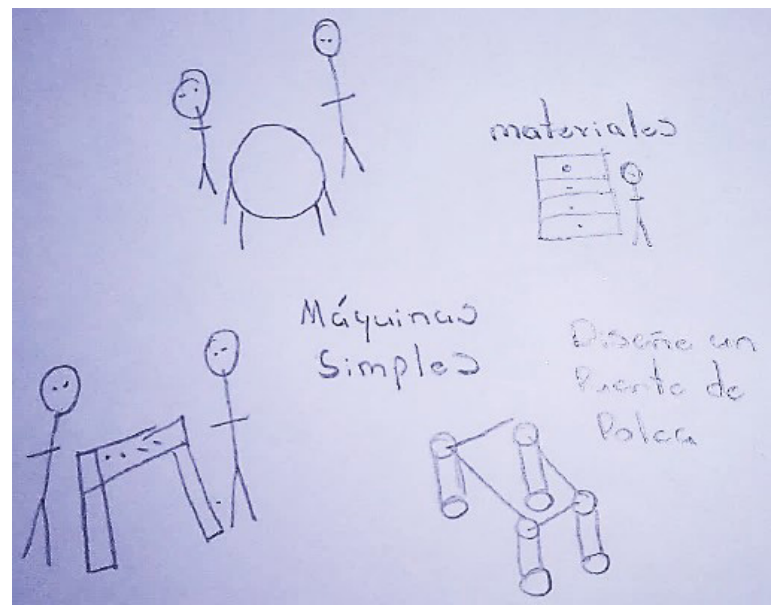

Figura 3. Ejemplo de representación categorizada como el modelo B de educación STEM.

Fuente: archivo de los autores.

El modelo C representa STEM como la enseñanza de las cuatro disciplinas que componen el acrónimo, si bien de manera disciplinar y separada. En estas representaciones las cuatro disciplinas están compartimentadas, no existiendo mención explícita a la integración disciplinar, pero en la que sí se representan los contenidos y se hace referencia a cada disciplina STEM (Figura 4):

"Es la enseñanza de las diversas disciplinas donde se utilizan diversos materiales."

"Es la incorporación de las C, T, I, M para provocar un aprendizaje más significativo y para que los estudiantes puedan ampliar y construir su conocimiento."
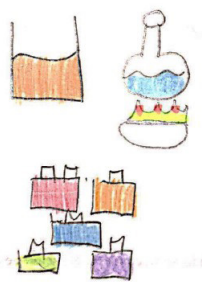
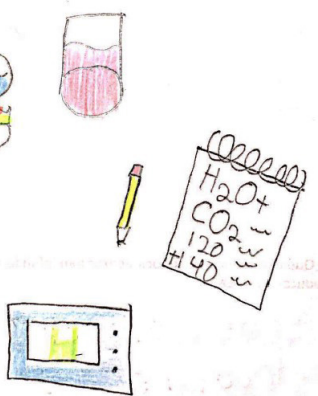

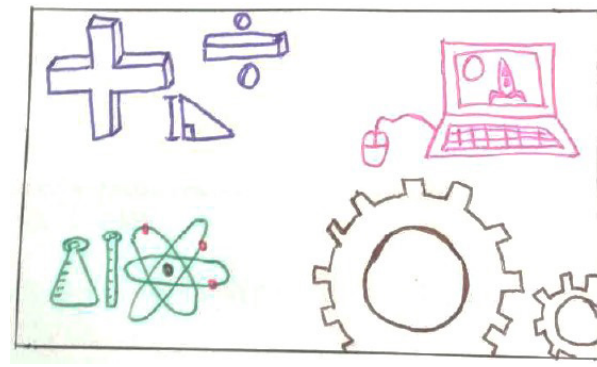

Figura 4. Ejemplo de representaciones categorizadas como el modelo $\mathrm{C}$ de educación STEM

Fuente: archivo de los autores. 
El modelo D concibe STEM como la resolución de problemas como principal foco didáctico para la formación de los estudiantes. En esta conceptualización, se representa parte de las disciplinas STEM y se menciona la importancia de hacer que el contenido STEM sea relevante para los estudiantes y les ayude en la resolución de problemas reales (Figura 5):

"Consiste en partir de problemas esenciales que surgen en el contexto en el que estamos insertos, para que los estudiantes puedan apropiarse de ellos y buscar soluciones de una manera crítica y responsable."

"Es usar la ciencia y otras disciplinas para solucionar problemas que se presentan en la realidad cercana de los niños y niñas. El dibujo representa un problema de contaminación de una fuente de agua y los estudiantes indagando al respecto. En una parte del dibujo, recolectan el agua de la laguna y en otra la estudian por medio de la tecnología y la ciencia."

"Incluye varias disciplinas para buscar solución a situaciones problemáticas cercanas al estudiante. Existen varias estructuras y formas de aplicar STEM dentro del aula, ya que las Ciencias pueden verse como una sola disciplina o puede incluirse varias áreas de saber."

"Los estudiantes pueden desarrollar el conocimiento científico desde las experiencias producidas en las diferentes situaciones que se planteen en el aula."
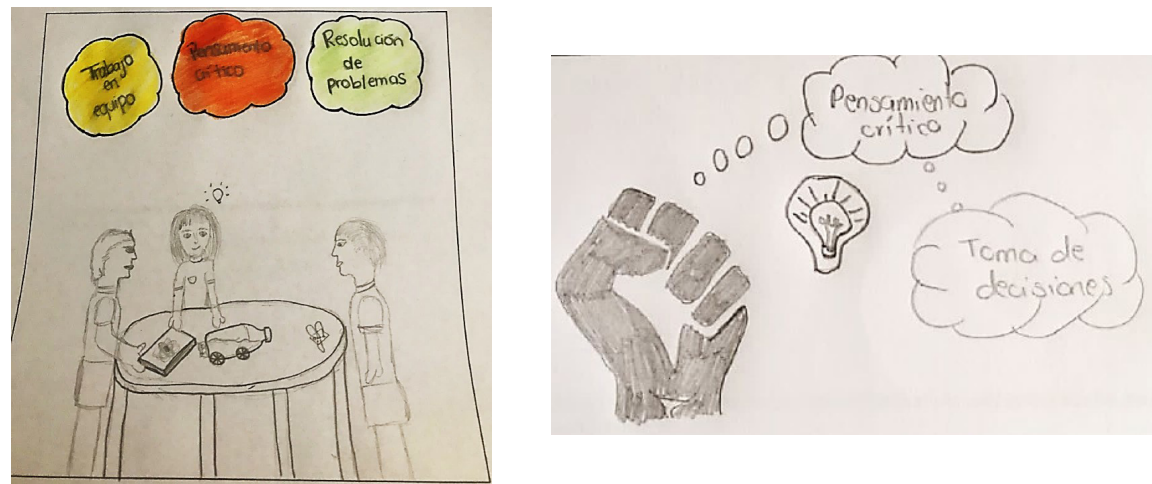

Figura 5. Ejemplo de representaciones categorizadas como el modelo D de educación STEM

Fuente: archivo de los autores.

El modelo E equivale a la enseñanza y el aprendizaje de una disciplina principal, generalmente la científica, incorporando aspectos de tecnología, ingeniería y/o matemáticas cuando sea pertinente. Esta conceptualización establece conexiones entre al menos dos disciplinas, sin embargo, no puede considerarse una integración dado que las disciplinas objeto de estudio no poseen el mismo estatus e importancia. En otras palabras, la T, E y M del acrónimo se emplean como un soporte para aprender la S (Figura 6):

"Para mi STEM es una forma de explicar la asignatura de Ciencias, por medio de la tecnología." 
"Es una educación diversificada, donde desde una misma línea se utilizan varias ramas de la ciencia para dar un conocimiento bien armado, si bien es cierto que muchas veces solo se utilizan una de las ramas y no todas."
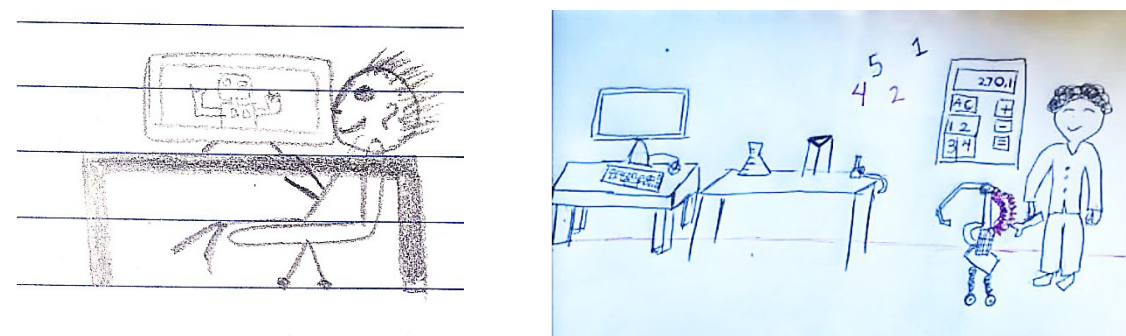

Figura 6. Ejemplo de representaciones categorizadas como el modelo E de educación STEM

Fuente: archivo de los autores.

Finalmente, el modelo $\mathrm{F}$ recoge aquellas conceptualizaciones que visualizan la educación STEM como la integración de las disciplinas. Estas conceptualizaciones representaban la confluencia entre las disciplinas STEM y sus contenidos, y aluden al uso de enfoques inter o transdisciplinares para establecer dicha integración (Figura 7):

"STEM es una enseñanza integrada que involucra varias asignaturas. Es verlo como un todo. Abandona el modelo tradicional y va más allá utilizando elementos más dinámicos. Busca fomentar habilidades, donde el niño tiene un papel importante y es quien dirige el proceso de enseñanza-aprendizaje. Considero que se busca generar un aprendizaje significativo."

"STEM en educación es el desarrollo de un conocimiento transversal en el que los contenidos de cada una de las ramas de ciencias, tecnología, ingeniería, y matemática trabajan de manera integrada y garantizan un aprendizaje contextualizado y significativo."

"Considero que es una educación sumamente integral, con una metodología innovadora en donde el estudiante es el actor de su propio aprendizaje, los docentes en este caso le dan un andamiaje con el fin de aclarar dudas y siempre acompañarlos en el proceso. En las lecciones se logra integrar la ciencia, la tecnología, la ingeniería y las matemáticas."
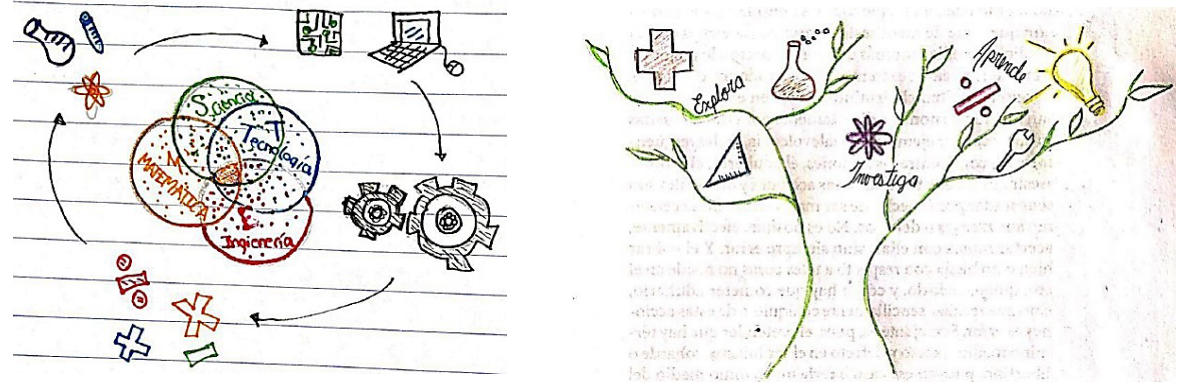

Figura 7. Ejemplo de representaciones categorizadas como el modelo F de educación STEM

Fuente: archivo de los autores. 
En cuanto a las experiencias que hayan podido influir en su conceptualización de STEM, todos los participantes han referido a una visita a un centro escolar, realizada durante el primer curso del Grado, en la que han podido observar de qué manera los docentes transponen didácticamente el enfoque educativo STEM:

"Las experiencias que he tenido han sido recorridos por escuelas que utilizan STEM dentro de sus metodologías de enseñanza. Durante estos recorridos observé los proyectos que estudian y realizan."

"En mi experiencia con la educación STEM fue la visita a una escuela que trabajaba con este sistema, en la cual se le otorgan roles a los niños para realizar trabajos de investigación que integraban ingeniería al tener que crear proyectos con materiales reciclados, matemáticas, ya que se debían hacer diferentes cálculos e incluso se involucraba la letra "A" de arte STEAM."

"Yo no conocía esta forma de trabajo hasta mi primer año de universidad en donde tuve la oportunidad de visitar dos centros escolares en donde se utilizaba."

"La visita de centros educativos en el curso de Pedagogía y Tecnología I me dejó ver el cómo se trabaja este modelo con los chicos de Primaria."

Asimismo, una minoría mencionó haber estudiado durante Educación Primaria con este modelo educativo, la influencia de lecturas y su profesorado, o el haber participado en eventos:

"Yo salí del colegio Isaac Martin, el cual en mis últimos años de colegio se basó en esta metodología, lo que me permitió conocer mejor la forma en la que se lleva a cabo."

"Probablemente de las lecturas y algunos comentarios de profesores de varios cursos."

"La visita a centros educativos. Otra experiencia es ser asistente de un docente en las Olimpiadas Nacionales de Robótica."

\section{2 ¿De qué manera cambia la conceptualización de los MCFI después de} participar en un programa de formación inicial interdisciplinar?

Una vez identificadas las conceptualizaciones sobre la educación STEM, se ha realizado un segundo análisis de las respuestas para categorizar todas las fuentes de datos en alguno de los seis modelos descritos anteriormente. La Tabla 3 recoge esta categorización del pretest y posttest.

Tabla 3. Concepciones de los MCFI sobre STEM

\begin{tabular}{lcccc}
\hline \multicolumn{1}{c}{ Modelos STEM } & \multicolumn{2}{c}{ Pretest } & \multicolumn{2}{c}{ Posttest } \\
\hline Modelo A - Acrónimo & $\mathrm{n}$ & $\%$ & $\mathrm{n}$ & $\%$ \\
Modelo B - Una disciplina & 5 & 22.7 & 0 & 0 \\
Modelo C - Disciplinas separadas & 2 & 9.1 & 0 & 0 \\
Modelo D - Relevancia & 8 & 36.4 & 8 & 36.4 \\
Modelo E - Conexión & 1 & 4.5 & 5 & 22.7 \\
Modelo F - Integración & 0 & 0 & 4 & 18.2 \\
\hline Fuente: elaboración propia. & 6 & 27.3 & 5 & 22.7 \\
\hline
\end{tabular}


Se observa que antes del programa de formación, predominaron las conceptualizaciones que refieren a STEM como cuatro disciplinas separadas entre las que no hay conexión ni integración (modelo C, 36.4\%). En segundo lugar, un 27.3\% representaron STEM como la integración de las cuatro disciplinas, siendo esta una de las conceptualizaciones más consensuadas actualmente en la literatura (modelo F, 27.3\%). Asimismo, cabe destacar un porcentaje relativamente alto de concepciones que reconocen STEM como un mero acrónimo (modelo A, 22.7\%). En términos generales, sumando las conceptualizaciones simplistas (modelos A, B, y C), se observa que un $68.2 \%$ de los participantes no conciben STEM de una manera adecuada ni coherente con las actuales definiciones que refieren a la conexión o integración curricular.

Tras la participación en el programa de formación, se han identificado cambios en las conceptualizaciones de los MCFI. En este sentido, cabe resaltar que ninguno de Ios participantes siguió conceptualizando STEM como un mero acrónimo (modelo A) o como una única disciplina (modelo B). El número de MCFI que han conceptualizado STEM como cuatro disciplinas separadas o compartimentadas se mantuvo (modelo C, $36.4 \%$ ). Sin embargo, aumentaron notablemente las conceptualizaciones de STEM como un enfoque innovador que se basa en la resolución de problemas para aumentar la relevancia del currículo escolar para el alumnado (modelo D, 22.7\%), así como las conceptualizaciones que refieren a STEM como la enseñanza y el aprendizaje de las Ciencias mediante el uso de otras disciplinas (modelo E, 18.2\%). Todos los participantes han referido al curso de formación como la principal fuente de experiencia que han influido en su conceptualización de STEM durante el posttest:

"Creo que para mi conceptualización de STEM, la combinación de elementos teóricos como prácticos ha sido fundamental. Ha sido un proceso de construcción largo que estoy segura de que aún no acaba."

"Creo que uno de los aspectos que más han influido en la conceptualización que tenía sobre STEM han sido las experiencias que hemos tenido a lo largo del curso, como en la realización de distintos experimentos que se pueden realizar para abarcar ciertos temas en educación primaria."

"Las experiencias obtenidas en el curso. Por ejemplo: la realización de un proyecto ingenieril ya que nunca había hecho uno. También la realización de un paracaídas casero."

Un análisis pormenorizado de los cambios en las concepciones de los MCFI (Figuras 8 y 9), revela que las conceptualizaciones de STEM como integración disciplinar (modelo F) no son fruto del programa de formación. Al contrario, parecería que los MCFI ya poseían dicha conceptualización inicialmente. Asimismo, se observa que las conceptualizaciones más propensas a ser modificadas son aquellas de un nivel más bajo (modelo A y B), si bien no han alcanzado niveles coherentes con la conceptualización de STEM de la literatura especializada (esto es, STEM como conexión o integración: modelos E y F). Finalmente, se observa que las conceptualizaciones de STEM como disciplinas compartimentadas (modelo $\mathrm{C}$ ) son las más difíciles de cambiar. De los 8 participantes con esta conceptualización al inicio del programa, apenas tres de ellos han modificado su concepción, pasando a un modelo de conexiones entre Ciencia y otras disciplinas (modelo E - participantes 9 y 12) o a un modelo que destaca la relevancia de las disciplinas mediante la resolución de problemas (modelo D - participante 22). 


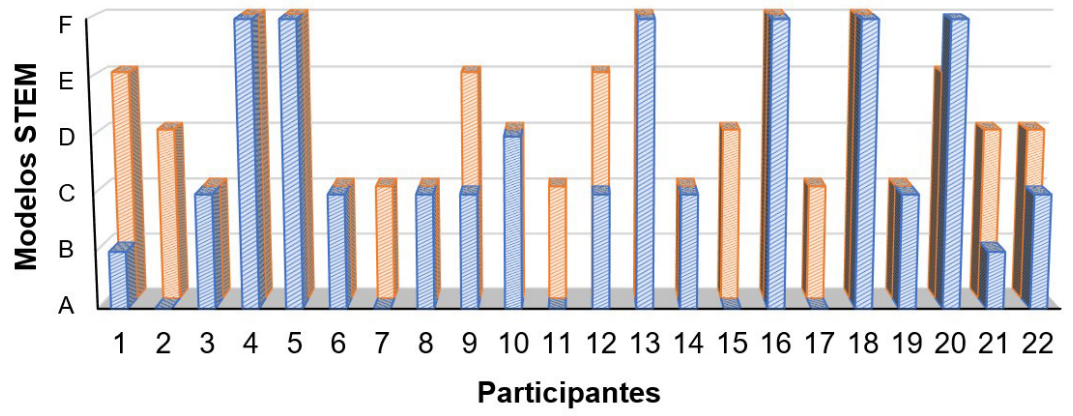

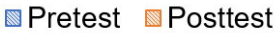

Figura 8. Concepciones sobre STEM durante el pretest y el posttest Fuente: elaboración propia.

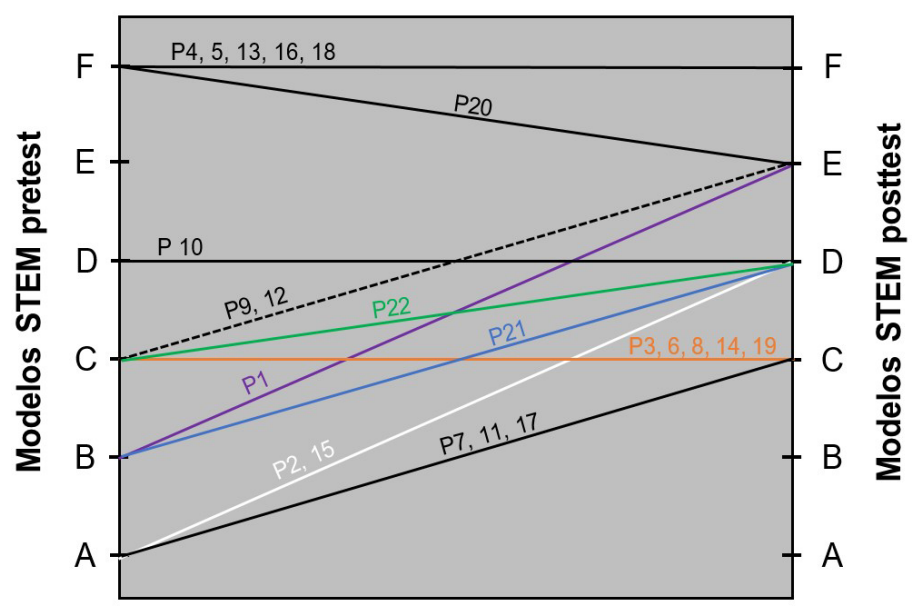

Figura 9. Cambio en las concepciones sobre STEM

Nota: $\mathrm{P}=$ participantes. Fuente: elaboración propia.

\section{Discusión}

La presente investigación tuvo por objeto identificar la forma en que un grupo de MCFI costarricenses conceptualizan la educación STEM y determinar en qué medida cambian sus conceptualizaciones tras la participación en un programa STEM de formación inicial. Los hallazgos revelan que los participantes de este estudio conceptualizan la educación STEM de manera muy distinta, lo que constituye un total de seis modelos que varían desde los más sencillos y limitados (STEM como (i) un acrónimo, (ii) una disciplina, generalmente, la científica, (iii) cuatro disciplinas compartimentadas) hasta modelos más complejos y consistentes con las definiciones recientes de STEM propuestas en la literatura especializada (STEM como (iv) resolución de problemas, (v) conexión entre ciencias y otras disciplinas, e (vi) integración de las cuatro disciplinas). Estos resultados son esperables, habida cuenta de la gran cantidad de definiciones y 
conceptualizaciones que coexisten en el ámbito educativo (Toma y García-Carmona, 2021). Asimismo, algunos de estos modelos coinciden con los identificados en estudios anteriores con maestros en formación inicial y docentes en activo (Çiftçi et al., 2020; Radloff y Guzey, 2016; Ring et al., 2017). Por otro lado, cabe destacar que el modelo E (conexión entre disciplinas) recuerdan a otros esfuerzos educativos, como el movimiento CTS que establece la relación entre la ciencia, el desarrollo tecnológico, y su impacto en los fenómenos sociales, y viceversa (Perales y Aguilera, 2020).

La gran mayoría de los MCFI revelaron conceptualizaciones muy simples y poco desarrolladas al inicio del programa de formación. Estos resultados concuerdan con la falta de consenso en la literatura acerca de qué es STEM, cómo se ha de conceptualizar teóricamente, y cómo ha de ser su implementación (Toma y García-Carmona, 2021). Cabe señalar que estos hallazgos coinciden con las visualizaciones teóricas sobre STEM de Bybee (2013), así como con las visiones identificadas en estudios previos. Así, Ring et al. (2017) y Dare et al. (2019) encontraron que las conceptualizaciones sobre STEM que predominan son las que refieren a STEM como un acrónimo o STEM como disciplinas separadas. Por otro lado, estos hallazgos difieren de los reportados por Radloff y Guzey (2016), que encontraron visiones más consistentes con conceptualizaciones conectadas o integradas en una muestra de docentes en formación inicial; si bien, estas diferencias podrían deberse a la diferencia entre los protocolos de recolección de datos, mucho más guiado en el caso de Radloff y Guzey (2016)².

Por otro lado, cabe destacar que, a pesar de las alusiones de los MCFI a visitas a centros escolares que trabajan con el enfoque STEM, lecturas de bibliografía STEM o inclusive haber participado en eventos STEM, no es de extrañar el predominio de conceptualizaciones simplistas, habida cuenta de que STEM constituye una "moda educativa" y que su uso en el ámbito educativo es abusivo y, a menudo, como un eslogan para atraer financiación o hacer propaganda de iniciativas y materiales educativo antiguos, rebautizados ahora como STEM (Toma y García-Carmona, 2021).

Al finalizar el programa de formación, las conceptualizaciones de los MCFI avanzaron hacia modelos más complejos (por ejemplo, modelo D). Sin embargo, ninguno de los MCFI con conceptualizaciones simplistas, esto es, STEM como un acrónimo, ha logrado avanzar hacia una conceptualización que aluda al establecimiento de conexiones entre disciplinas o la integración de estas. Del mismo modo, el número de MCFI que mantuvieron una conceptualización de STEM como cuatro disciplinas compartimentadas (modelo C) se mantuvo similar después de la intervención. Por lo tanto, se observan tres tendencias principales. En primer lugar, las conceptualizaciones iniciales más susceptibles al cambio son las pertenecientes a STEM como un acrónimo (modelo A). Así, los participantes transitan de esta conceptualización a STEM como disciplinas separadas (modelo C) o STEM como relevancia (modelo D). Por otro lado, las visiones de STEM como disciplinas separadas (modelo $\mathrm{C}$ ), que corresponde con la acepción original del acrónimo STEM acuñada a principios de la década de los años 90 (Toma y García-Carmona, 2021), parecerían ser las más difíciles de modificar; siendo que estos resultados no concuerdan con estudios pasados (p.ej. Ring et al., 2017).

\footnotetext{
${ }^{2}$ En su protocolo, se identifican preguntas que aluden a STEM como un método de instrucción, a la conexión entre las disciplinas STEM, o a la instrucción guiada de "Dibuja un diagrama de cómo lo visualizas utilizando las letras S-T-E-M, así como de cómo están conectadas" (preguntas 8,9 y 11; p. 773, énfasis añadido).
} 
Finalmente, la visión de STEM como relevancia (modelo D) parecería ser la más fácil de interiorizar por docentes en formación inicial tras su participación en el programa de formación propuesto. En otras palabras, la intervención ha resultado útil en resaltar la necesidad de hacer que el contenido STEM sea relevante para los estudiantes y útil para la resolución de problemas cotidianos; sin embargo, no ha conseguido transmitir la conceptualización de que dicha relevancia se podría fomentar, según concepciones contemporáneas de STEM (p.ej. Johnson et al., 2016; Kelley y Knowles, 2016; Martín-Páez et al., 2019)technology, engineering, and mathematics (STEM, mediante la conexión y/o integración de las cuatro disciplinas que componen el acrónimo.

Por otro lado, aunque varios MCFI poseían una visión de STEM coherente con las definiciones contemporáneas (modelo F), cabe reflexionar en qué medida dichas conceptualizaciones realmente reflejan las características de un enfoque STEM interdisciplinar auténtico. En este sentido, es probable que, aunque algunos MCFI concibieran STEM como la integración de cuatro disciplinas, esta conceptualización resultara menos informada si se hubiera profundizado en la manera en la que éstos conciben la tecnología, la ingeniería, o la forma en la que se han de integrar estas disciplinas. Este aspecto, no abordado en esta investigación, justifica la realización de futuros estudios que profundicen en las conceptualizaciones de STEM por parte de los educadores, solicitando ejemplos específicos de cada modelo (p.ej. ¿Qué significa integrar cuatro disciplinas? ¿De qué manera se concibe la tecnología? ¿Qué es la ingeniería y cómo se emplea en niveles elementales del sistema educativo?). Por lo tanto, estos resultados ofrecen un apoyo parcial a la afirmación de que las conceptualizaciones de STEM pueden ser fácilmente modificadas a través de programas de desarrollo profesional (cf. Herro y Quigley, 2017; Ring et al., 2017).

Estos resultados poseen importantes implicaciones. A nivel de investigación, refuerzan las demandas por alcanzar un consenso sobre cómo definir STEM y de qué manera transponerlo a la práctica educativa, al mismo tiempo que arrojan dudas sobre la idoneidad de un enfoque de estas características dada su difícil conceptualización y concreción educativa (García-Carmona, 2020; Toma y García-Carmona, 2021). Por otro lado, en tanto se pretenda que el enfoque STEM suponga una mejora de las prácticas pedagógicas del futuro profesorado de ciencias, los hallazgos de este estudio destacan la importancia de abordar durante su formación inicial sus modelos mentales de la educación STEM, estableciendo espacios de reflexión y de práctica educativa que permitan reconsiderar sus conceptualizaciones. Este aspecto, cuya consecución resulta no poco difícil, tal y como se muestra en esta investigación, resulta fundamental si se desea fomentar que los docentes en formación inicial adopten un enfoque STEM integrado durante su labor docente futura. Por lo tanto, es importante diagnosticar la diversidad de conceptualizaciones existentes y desarrollar medidas educativas enfocadas a enriquecerlas con modelos STEM más completos, tales como la integración de las cuatro disciplinas que componen dicho acrónimo. De lo contrario, STEM difícilmente supondrá una mejora de la educación científica y continuará siendo un marco deficitario para la renovación pedagógica en la didáctica de las ciencias experimentales (Zeidler, 2016). 


\subsection{Limitaciones}

Los resultados de esta investigación deben interpretarse a la luz de las siguientes dos limitaciones. En primer lugar, el contexto de la intervención, así como el tamaño muestral limitan la generalización de estos resultados. Específicamente, el programa de formación inicial se implementó de manera virtual mediante sesiones sincrónicas y asincrónicas, debido a la pandemia del COVID-19 y las medidas de confinamiento domiciliario. Este aspecto limitó claramente la interacción profesorado-alumnado y alumnado-alumnado, especialmente durante las sesiones de indagación y diseño ingenieril. Asimismo, el contexto COVID-19 afectó al tiempo dedicado durante el programa a cada una de las fases del modelo STEM por motivos de disponibilidad de recursos. El modelo adoptado, basado en Toma (2020b), otorga la misma prioridad a la primera (aprendizaje de conocimientos mediante indagaciones escolares) y segunda fase (aplicación de conocimientos mediante retos de diseño ingenieril). Sin embargo, en el presente estudio se dedicaron más sesiones a la primera fase a que las experiencias propuestas requerían de menos recursos y materiales que los retos de diseño ingenieril.

No obstante, estos resultados sí han permitido identificar conceptualizaciones en población costarricense que concuerdan con la literatura internacional, y resaltar algunos patrones relacionados con la dificultad de modificar dichas conceptualizaciones, especialmente las que al inicio de la intervención fueron muy simples y poco desarrolladas. La segunda limitación está relacionada con las respuestas escritas del protocolo de elicitación. De este modo, si bien la categorización de las representaciones resultó operativa, alcanzándose un alto acuerdo interevaluador, una minoría de las respuestas escritas de los MCFI podrían reflejar varias categorías. Por ejemplo, la primera parte de la siguiente respuesta parecería aludir al modelo D de STEM (relevancia), sin embargo, la segunda parte se relaciona indudablemente con el modelo F (integración): "Considero que es una educación sumamente integral, con una metodología innovadora en donde el estudiante es el actor de su propio aprendizaje, los docentes en este caso le dan un andamiaje con el fin de aclarar dudas y siempre acompañarlos en el proceso. En las lecciones se logra integrar la ciencia, la tecnología, la ingeniería y las matemáticas." Este aspecto podría señalar que, aunque el discurso común sobre STEM se haya interiorizado (p.ej. STEM como integración disciplinar), la operativización de dicha conceptualización sea aún muy simplista, lo que subraya las dudas de su viabilidad (Toma y García-Carmona, 2021).

\section{Agradecimientos}

Al alumnado matriculado en la asignatura FD0516 Ciencias en la Educación Primaria II de la Facultad de Educación de la Universidad de Costa Rica, por su compromiso y participación en el programa de formación a pesar de las complicadas circunstancias causadas por la pandemia del COVID-19. 


\section{Referencias}

Breiner, J. M., Harkness, S. S., Johnson, C. C. \& Koehler, C. M. (2012). What is STEM? A discussion about conceptions of STEM in education and partnerships. School Science and Mathematics, 112(1), 3-11. https://doi.org/10.1111/j.1949-8594.2011.00109.x

Bybee, R. W. (2013). The case for STEM education. Challenges and opportunities. NSTA press.

Capps, D. K., Crawford, B. A. \& Constas, M. A. (2012). A review of empirical literature on inquiry professional development: Alignment with best practices and a critique of the findings. Journal of Science TeacherEducation, 23(3), 291-318. https://doi.org/10.1007/s10972-012-9275-2

Çiftçi, A., Topçu, M. S. \& Foulk, J. A. (2020). Pre-service early childhood teachers' views on STEM education and their STEM teaching practices. Research in Science and Technological Education, 00(00), 1-27. https://doi.org/10.1080/02635143.2020.1784125

Couso, D. y Grimalt-Álvaro, C. (2020). ¿Qué es STEM y STEAM y por qué ponerse a ello? Aula de Innovación Educativa, 290, 71-72.

Crawford, B. A. (2014). From inquiry to scientific practices in the science classroom. In N. G. Lederman \& S. K. Abell (Eds.), Handbook of research on science education, Volume II (pp. 515541). Routledge.

Cunningham, C. M. (2018). Engineering in elementary STEM education: Curriculum design, instruction, learning, and assessment. Teachers College Press and Museum of Science Driveway.

Dare, E. A., Ring-Whalen, E. A. \& Roehrig, G. H. (2019). Creating a continuum of STEM models: Exploring how K-12 science teachers conceptualize STEM education. International Journal of Science Education, 41(12), 1701-1720. https://doi.org/10.1080/09500693.2019.1638531

Domènech-Casal, J. (2019). STEM: Oportunidades y retos desde la Enseñanza de las Ciencias. Universitas Tarraconensis. Revista de Ciències de l'Educació, 1(2), 154. https://doi. org/10.17345/ute.2019.2.2646

García-Carmona, A. (2020). STEAM, ¿una nueva distracción para la enseñanza de la ciencia? Ápice. Revista de Educación Cientifica, 4(2), 35-50. https://doi.org/10.17979/arec.2020.4.2.6533

Herro, D. \& Quigley, C. (2017). Exploring teachers' perceptions of STEAM teaching through professional development: implications for teacher educators. Professional Development in Education, 43(3), 416-438. https://doi.org/10.1080/19415257.2016.1205507

Herron, M. D. (1971). The nature of scientific enquiry. The School Review, 79(2), 171-212. http://link. springer.com/10.1057/9781137389831

Honey, M., Pearson, C. \& Schweingruber, A. (2014). STEM integration in K-12 education: Status, prospects, and an agenda for research. The National Academies Press.

Hsieh, H.-F. \& Shannon, S. E. (2005). Three Approaches to Qualitative Content Analysis. Qualitative Health Research, 15(9), 1277-1288. https://doi.org/10.1177/1049732305276687

Johnson, C. C., Peters-Burton, E. E. \& Moore, T. J. (2016). STEM road map: A framework for integrated STEM education. Routledge Taylor \& Francis Group.

Kelley, T. R. \& Knowles, J. G. (2016). A conceptual framework for integrated STEM education. International Journal of STEM Education, 3(11), 1-11. https://doi.org/10.1186/s40594-016-0046-z

Margot, K. C. \& Kettler, T. (2019). Teachers' perception of STEM integration and education: a systematic literature review. International Journal of STEM Education, 6(1). https://doi.org/10.1186/ s40594-018-0151-2

Martin-Hansen, L. (2002). Defining inquiry. The Science Teacher, 69(2), 34-37.

Martín-Páez, T., Aguilera, D., Perales-Palacios, F. J. \& Vílchez-González, J. M. (2019). What are we talking about when we talk about STEM education? A review of literature. Science Education, 103(4), 799-822. https://doi.org/10.1002/sce.21522

Pedaste, M., Mäeots, M., Siiman, L. A., de Jong, T., van Riesen, S. A. N., Kamp, E. T., Manoli, C. C., Zacharia, Z. C. \& Tsourlidaki, E. (2015). Phases of inquiry-based learning: Definitions and the inquiry cycle. Educational Research Review, 14, 47-61. https://doi.org/10.1016/j. edurev.2015.02.003 
Perales, F. J. y Aguilera, D. (2020). Ciencia-Tecnología-Sociedad vs. STEM: ¿evolución, revolución o disyunción? Ápice. Revista de Educación Científica, 4(1), 1-15. https://doi.org/10.17979/ arec.2020.4.1.5826

Radloff, J. \& Guzey, S. (2016). Investigating Preservice STEM Teacher Conceptions of STEM Education. Journal of Science Education and Technology, 25(5), 759-774. https://doi.org/10.1007/ s10956-016-9633-5

Ring, E. A., Dare, E. A., Crotty, E. A. \& Roehrig, G. H. (2017). The evolution of teacher conceptions of STEM education throughout an intensive professional development experience. Journal of Science Teacher Education, 28(5), 444-467. https://doi.org/10.1080/104656 0X.2017.1356671

Roehrig, G. H. \& Luft, J. A. (2004). Constraints experienced by beginning secondary science teachers in implementing scientific inquiry lessons. International Journal of Science Education, 26(1), 3-24. https://doi.org/10.1080/0950069022000070261

Sanders, M. (2009). STEM, STEM education, STEM mania. Technology Teacher, 68(4), 20-26.

Toma, R. B. (2020a). Integrando la programación computacional en el enfoque STEM: un ejemplo sobre la calidad del agua [Integrating computational programming into the STEM approach: an example on water quality] (I. M. Greca \& J. Á. Meneses-Villagrá (eds.)). Dextra Editorial S.L.

Toma, R. B. (2020b). STEM education in elementary grades: Design of an effective framework for imporving attitudes towards school science. University of Burgos.

Toma, R. B. \& García-Carmona, A. (2021). «De STEM nos gusta todo menos STEM». Análisis crítico de una tendencia educativa de moda. Enseñanza de Las Ciencias. Revista de Investigación y Experiencias Didácticas, 39(1), 65.

Toma, R. B. \& Greca, I. M. (2018). The effect of integrative STEM instruction on elementary students' attitudes toward science. EURASIA Journal of Mathematics, Science \& Technology Education, 14(4), 1383-1395. https://doi.org/10.29333/ejmste/83676

Vergara, C. y Cofré, H. (2012). La Indagación Científica: Un concepto esquivo, pero necesario. Revista Chilena de Educación Científica, 11(1), 30-38.

Zeidler, D. L. (2016). STEM education: A deficit framework for the twenty first century? A sociocultural socioscientific response. Cultural Studies of Science Education, 11(1), 11-26. https://doi. org/10.1007/s11422-014-9578-z

Cómo citar en APA:

Toma, R. B. y Retana-Alvarado, D. A. (2021). Mejora de las concepciones de maestros en formación de la educación STEM. Revista Iberoamericana de Educación, 87(1), 15-33. https://doi. org/10.35362/rie8714538 